\title{
Weakly prime ideals issued from an amalgamated algebra
}

\author{
Najib Mahdou${ }^{1}$ (D), Moutu Abdou Salam Moutui*2 (D), Youssef Zahir ${ }^{1}$ (i) \\ ${ }^{1}$ Modelling and Mathematical Structures Laboratory, Department of Mathematics, Faculty of Science and \\ Technology of Fez, Box 2202, University S.M. Ben Abdellah Fez, Morocco \\ ${ }^{2}$ Department of Mathematics, College of science, King Faisal University, P. O. Box 400, Al-Ahsaa 31982, \\ Saudi Arabia
}

\begin{abstract}
Let $R$ be a commutative ring with identity. A proper ideal $P$ is said to be weakly prime ideal of $R$ if for every $0 \neq a b \in P$ where $a, b \in R$, implies $a \in P$ or $b \in P$. The notion of weakly prime ideal was introduced by Anderson et al. in [Weakly prime ideals, Houston J. Math., 2003] as a generalization of prime ideals. The purpose of this paper is to study the form of weakly prime ideals of amalgamation of $A$ with $B$ along $J$ with respect to $f$ (denoted by $A \bowtie^{f} J$ ), introduced and studied by D'Anna et al. in [Amalgamated algebras along an ideal, Commutative Algebra and Its Applications, 2009]. Our results provide new techniques for the construction of new original examples of weakly prime ideals. Furthermore, as an application of our results, we provide an upper bound for the weakly Krull dimension of amalgamation.
\end{abstract}

Mathematics Subject Classification (2010). 13F05, 13A15, 13E05, 13F20, 13C10, 13C11, 13F30, 13D05

Keywords. Amalgamated algebra, weakly prime ideal, weakly Krull dimension, amalgamated duplication

\section{Introduction}

All rings considered below are assumed to be commutative with identity and all modules are assumed to be unital. Among the many generalizations of the prime ideals in the literature, we find the following due to D.D. Anderson and E. Smith in [3], derived from the study of factorization in commutative rings with zero-divisors (see [1] for more details). A proper ideal $P$ of $R$ is called weakly prime ideal if $0 \neq a b \in P$ where $a, b \in R$ implies $a \in P$ or $b \in P$. Every prime ideal of $R$ is weakly prime. However, the converse is not true. For instance, $\{0\}$ is always weakly prime of $R$, and it is prime if and only if $R$ is an integral domain. Note that every proper ideal in a ring $R$ is weakly prime ideal if and only if either $R$ is a quasilocal ring (possibly a field) whose maximal ideal is square 0 , or $R$ is product of two field [3, Theorem 8]. They studied the form of weakly prime ideals in a

\footnotetext{
*Corresponding Author.

Email addresses: mahdou@hotmail.com (N. Mahdou), mmoutui@kfu.edu.sa (M. A. S. Moutui), youssef.zahir@usmba.ac.ma (Y. Zahir)

Received: 24.04.2019; Accepted: 27.08.2019
} 
decomposable rings $R$ (i.e., there exist nontrivial rings $R_{1}$ and $R_{2}$ such that $R=R_{1} \times R_{2}$ ) and showed that if $P$ is weakly prime ideal of $R$, either $P=0$ or $P$ is prime ideal. A number of results concerning weakly prime ideals and examples of weakly prime ideals are given in [3]. On the other hand, they showed how to construct examples of weakly prime ideals using the trivial ring extension. In 2016, Badawi defined in [5] the notion of weakly semiprime ideal considered as generalization of semiprime ideals. Recall that an ideal $I$ of $R$ is said to be semiprime if, whenever $x^{2} \in I$ for element $x \in R$ we have $x \in I$. A proper ideal $P$ of a ring $R$ is called a weakly semiprime ideal if $a \in R$ and $0 \neq a^{2} \in P$ implies $a \in P$. Clearly, every weakly prime ideal of $R$ is weakly semiprime. However, the converse is not true. For instance, let $R=Z_{16}$ and $I=\{0,8\}$ be an ideal of $R$. By definition, $I$ is a weakly semiprime ideal of $R$ which is not weakly prime.

Let $A$ and $B$ be two rings with unity, let $J$ be an ideal of $B$ and let $f: A \longrightarrow B$ be a ring homomorphism. In this setting, we consider the following subring of $A \times B$ :

$$
A \bowtie^{f} J:=\{(a, f(a)+j) \in A \times B \mid a \in A, j \in J\}
$$

is called the amalgamation of $A$ and $B$ along $J$ with respect to $f$. This construction is a generalization of the amalgamated duplication of a ring along an ideal denoted $A \bowtie I$ (introduced and studied by D'Anna and Fontana in [10]). In [8,9], D'Anna, Finocchiaro and Fontana introduced the more general context of amalgamations. They have studied these constructions in the frame of pullbacks which allowed them to establish numerous results on the transfer of various ideal and ring-theoretic properties from $A$ and $f(A)+J$ to $A \bowtie^{f} J$. The concept of amalgamation is an important and an interesting concept that received a considerable attention by well-known established algebraists. The interest of amalgamations resides in their ability to cover basic constructions in commutative algebra, including classical pullbacks and trivial ring extensions. Moreover, other classical constructions (such as $A+X B[X], A+X B[[X]]$ and the $D+M$ constructions) can be studied as particular cases of the amalgamation ([8, Examples 2.5 and 2.6]) and other classical constructions, such as the CPI extensions (in the sense of Boisen and Sheldon [6]) are strictly related to it ([8, Example 2.7 and Remark 2.8]). In [8], the authors studied the basic properties of this construction (e.g., characterizations for $A \bowtie^{f} J$ to be a Noetherian ring, an integral domain, a reduced ring) and they characterized those distinguished pullbacks that can be expressed as an amalgamation. Moreover, in [9], they pursued the investigation on the structure of the rings of the form $A \bowtie^{f} J$, with particular attention to the prime spectrum, to the chain properties and to the Krull dimension. Amalgamation rings have been studied extensively, often because of their usefulness in constructing new classes of examples of rings satisfying various properties (for instance see $[2,7,12,13])$.

In this paper, we pursue the investigation on the structure of the ring of the form $A \bowtie^{f} J$, with a particular attention to the form of weakly prime ideals and to weakly Krull dimension introduced and studied in [14]. The weakly krull dimension of $R$ denoted by $w-\operatorname{dim}(R)$, is the maximum number $n \in \mathbb{N}$ such that there is a chain of weakly prime ideals $P_{0} \subset P_{1} \subset \ldots \subset P_{n}$ of length $n$ in $R$. For a ring $R$, we denote by $N i l p(R)$, the set of all nilpotent elements of $R$.

\section{Weakly prime ideals of amalgamations}

To avoid unnecessary repetition, let us fix notation for the rest of the paper. Let $(A, B)$ be a pair of rings, $f: A \rightarrow B$ be a ring homomorphism and $J$ be an ideal of $B$. All along this paper, $A \bowtie^{f} J$ will denote the amalgamation of $A$ and $B$ along $J$ with respect to $f$. Let $I$ be an ideal of $A$ and $K$ be an ideal of $f(A)+J$. Notice that $I \bowtie^{f} J:=\{(i, f(i)+j) / i \in I, j \in J\}$ and $\bar{K}^{f}:=\{(a, f(a)+j) / a \in A, j \in J, f(a)+j \in K\}$ 
are ideals of $A \bowtie^{f} J$. Our first result is a characterization of weakly prime ideals of the form $I \bowtie^{f} J$ and $\bar{K}^{f}$ of amalgamation $A \bowtie^{f} J$.

Theorem 2.1. Under the above notations, the following statements hold:

(1) $I \bowtie^{f} J$ is a weakly prime ideal of $A \bowtie^{f} J$ if and only if $I$ is a weakly prime ideal of $A$ and for $a, b \in A$ with $a b=0$, but $a, b \in A \backslash I$, then $f(a) j+f(b) i+i j=0$ for every $i, j \in J$.

(2) $\bar{K}^{f}$ is a weakly prime ideal of $A \bowtie^{f} J$ if and only if $K$ is a weakly prime ideal of $f(A)+J$ and when $f(a)+j, f(b)+k \notin K$ with $a, b \in A, j, k \in J$ and $(f(a)+$ $j)(f(b)+k)=0$, then $a b=0$.

Proof. (1) Assume that $I \bowtie^{f} J$ is a weakly prime ideal of $A \bowtie^{f} J$. Let $a b \in I-\{0\}$ where $a, b \in A$. Then $(a, f(a))(b, f(b)) \in I \bowtie^{f} J-\{(0,0)\}$ and so $(a, f(a)) \in I \bowtie^{f} J$ or $(b, f(b)) \in I \bowtie^{f} J$. Hence $a \in I$ or $b \in I$. Now, we claim that if $a, b \notin I$ with $a b=0$, then $f(a) j+f(b) i+i j=0$ for every $i, j \in J$. Deny. There exist $i, j \in J$ such that $f(a) j+f(b) i+i j \neq 0$ and so $(0,0) \neq(a, f(a)+i)(b, f(b)+j)=(a b, f(a b)+f(a) j+f(b) i+$ $i j)=(0, f(a) j+f(b) i+i j) \in I \bowtie^{f} J$, which is a contradiction since $(a, f(a)+i) \notin I \bowtie^{f} J$, $(b, f(b)+j) \notin I \bowtie^{f} J$ and $I \bowtie^{f} J$ is a weakly prime ideal of $A \bowtie^{f} J$.

Conversely, let $(a, f(a)+i)(b, f(b)+j)=(a b, f(a b)+f(a) j+f(b) i+i j) \in I \bowtie^{f} J-\{(0,0)\}$. Hence, $a b \in I$. Two cases are then possible:

Case 1: $a b \neq 0$.

Hence, $a \in I$ or $b \in I$ since $I$ is a weakly prime ideal of $A$. So, $(a, f(a)+i) \in I \bowtie^{f} J$ or $(b, f(b)+j) \in I \bowtie^{f} J$.

Case 2: $a b=0$.

We claim that $a \in I$ or $b \in I$. Deny. We have $f(a) j+f(b) i+i j=0$, contradiction with $(a, f(a)+i)(b, f(b)+j) \neq(0,0)$.

Therefore, in all cases, $a \in I$ or $b \in I$ and so $(a, f(a)+i) \in I \bowtie^{f} J$ or $(b, f(b)+j) \in I \bowtie^{f} J$, as desired.

(2) Assume that $\bar{K}^{f}$ is a weakly prime ideal of $A \bowtie^{f} J$. We claim that $K$ is a weakly prime ideal of $f(A)+J$. Indeed, let $x y \in K \backslash\{0\}$ with $x, y \in f(A)+J$. Then $x=f(a)+j$ and $y=f(b)+k$ for some $a, b \in A$ and $j, k \in J$. Clearly, $(a, f(a)+j)$ and $(b, f(b)+k)$ are nonzero elements of $\bar{K}^{f}$. Therefore, $(a, f(a)+j)(b, f(b)+k)=(a b,(f(a)+j)(f(b)+k)) \in$ $\bar{K}^{f}-\{(0,0)\}$ which is a weakly prime ideal of $A \bowtie^{f} J$. Consequently, $(a, f(a)+j) \in \bar{K}^{f}$ or $(b, f(b)+j) \in \bar{K}^{f}$, making $f(a)+j \in K$ or $f(b)+k \in K$. Hence, $K$ is a weakly prime ideal of $f(A)+J$. Now, let $f(a)+j, f(b)+k \notin K$ with $(f(a)+j)(f(b)+k)=0$. We claim that $a b=0$. Deny, $(a, f(a)+j)(b, f(b)+k)=(a b, 0) \in \bar{K}^{f}-\{(0,0)\}$, which is weakly prime ideal of $A \bowtie^{f} J$, a contradiction since $(a, f(a)+j) \notin \bar{K}^{f}$ and $(b, f(b)+k) \notin \bar{K}^{f}$. Hence, $a b=0$.

Conversely, let $(a, f(a)+j)(b, f(b)+k)=(a b,(f(a)+j)(f(b)+k)) \in \bar{K}^{f}-\{(0,0)\}$. So, $(f(a)+j)(f(b)+k)$ is an element of $K$ which is weakly prime ideal of $f(A)+J$. Two cases are then possible:

Case 1: $(f(a)+j)(f(b)+k) \neq 0$, then $f(a)+j \in K$ or $f(b)+k \in K$. Hence, $(a, f(a)+j) \in$ $\bar{K}^{f}$ or $(b, f(b)+k) \in \bar{K}^{f}$, as desired.

Case 2: $(f(a)+j)(f(b)+k)=0$.

We claim that $f(a)+j \in K$ or $f(b)+k \in K$. Deny, it follows that $a b=0$, which is absurd since $(a b,(f(a)+j)(f(b)+k)) \in \bar{K}^{f}-\{(0,0)\}$. Hence $(a, f(a)+j) \in \bar{K}^{f}$ or $(b, f(b)+k) \in \bar{K}^{f}$, making $\bar{K}^{f}$ a weakly prime ideal, as desired. Therefore, in all cases, $\bar{K}^{f}$ a weakly prime ideal. 
Next, we show how one may use Theorem 2.1 to construct original examples of weakly prime ideals of the form $I \bowtie^{f} J$ of amalgamation $A \bowtie^{f} J$ which are not primes. Recall that for a ring $A$ and an $A$-module $E$, the trivial ring extension of $A$ by $E$ (also called idealization of $E$ over $A$ ) is the ring $R:=A \propto E$ whose underlying group is $A \times E$ with multiplication given by $(a, e)\left(a^{\prime}, e^{\prime}\right)=\left(a a^{\prime}, a e^{\prime}+e a^{\prime}\right)$. Also recall that $\operatorname{Spec}(R)=\{P \propto$ $E \mid P \in \operatorname{Spec}(A)\}$ ([4, Theorem 3.1]).

Example 2.2. Let $A_{1}:=K[X, Y] /\left(X^{2} Y^{2}\right)$ be a ring, where $K$ is a field and $E:=$ $K[X, Y] /(X, Y)=K$ as an $A_{1}$-module. Consider $A:=A_{1} \propto E$ be the trivial ring extension of $A_{1}$ by $E$ and $B:=A_{1}$ be a ring. Notice that by [3, Example 20], $I:=\overline{0} \propto E$ is a weakly prime ideal of $A$. Let $f: A \rightarrow B$ be a surjective ring homomorphism and $J:=(X Y) /\left(X^{2} Y^{2}\right)$ be a proper ideal of $B$. Then:

(1) $I \bowtie^{f} J$ is a weakly prime ideal of $A \bowtie^{f} J$.

(2) $I \bowtie^{f} J$ is not a prime ideal of $A \bowtie^{f} J$.

Proof. (1) Notice that by [3, Example 20], $I:=\overline{0} \propto E$ is a weakly prime ideal of $A$. Assume that there exist $u=(\bar{x}, e), v=(\bar{y}, f) \notin I=\overline{0} \propto E, \bar{x}, \bar{y} \in A_{1}$ and $e, f \in E$ such that $u v=(\overline{0}, 0)$. Hence, $\bar{x} \bar{y}=\overline{0}$. Since $I$ is weakly prime ideal, we get $\bar{x} \in A n n(K)=0$ and $\bar{y} \in \operatorname{Ann}(K)=0$, contradiction. By Theorem 2.1(1), $I \bowtie^{f} J$ is weakly prime.

(2) By [9, Proposition $2.6(3)], I \bowtie^{f} J$ is not a prime ideal of $A \bowtie^{f} J$ since $I:=\overline{0} \propto E$ is not a prime ideal of $A$ (as $\overline{0}$ is not prime ideal of $A_{1}$ ).

The following corollary is an immediate consequence of Theorem 2.1 on the transfer of weakly semiprime property to amalgamations.

Corollary 2.3. Under the above notations, the following statements hold:

(1) Let $I$ be an ideal of $A$. Then $I \bowtie^{f} J$ is a weakly semiprime ideal of $A \bowtie^{f} J$ if and only if $I$ is a weakly semiprime ideal of $A$ and $a \in A \backslash I$ with $a^{2}=0$, then $2 f(a) j+j^{2}=0$ for each $j \in J$.

(2) Let $K$ be an ideal of $f(A)+J$. Then $\bar{K}^{f}$ is a weakly semiprime ideal of $A \bowtie^{f} J$ if and only if $K$ is a weakly semiprime ideal of $f(A)+J$ and when $f(a)+j \notin K$ with $a \in A, j \in J$ and $(f(a)+j)^{2}=0$, then $a^{2}=0$.

The next result is a particular case of assertion (1) of Theorem 2.1.

Corollary 2.4. Let $(A, M)$ be a quasilocal ring, $B$ be a ring, $f: A \longrightarrow B$ be a ring homomorphism and $J$ be a proper ideal of $B$ such that $J \subseteq f(A)$ and $f(M) J=0$. Consider an ideal $I$ of $A$. Then $I \bowtie^{f} J$ is a weakly prime ideal of $A \bowtie^{f} J$ if and only if $I$ is a weakly prime ideal of $A$.

Proof. By Theorem 2.1, it suffice to prove that, if $a, b \in A \backslash I$ with $a b=0$ then $f(a) j+$ $f(b) i+i j=0$ for each $i, j \in J$. Indeed, if $a b=0$ and $a \notin I$ and $b \notin I$ then $a \in M$ and $b \in M$. Then $f(a) j=f(b) i=0$ since $f(M) J=0$. Now we claim that $i j=0$. There exist $c \in M$ such that $i=f(c)$ since $J \subseteq f(A)$. Deny, there exist $d \in A$ such that $c d=1$ and $1=f(c d)=f(c) f(d)=i . f(d) \in J$. Then $J=B$, contradiction. Therefore, $f(a) j+f(b) i+i j=0$ for each $i, j \in J$, as desired.

Corollary 2.4 allows us to construct new original classes of weakly prime ideals.

Example 2.5. Let $(B, M)$ be a quasilocal ring with $M^{2}=0, A:=B \propto E$ is the trivial ring extension of $B$ by $E$, where $E$ is a semisimple $B$-module, $f: A \longrightarrow B$ is a surjective ring homomorphism and $J:=M$ is the maximal ideal of $B$. Consider $I:=I^{\prime} \propto E$, an ideal of $A$, where $I^{\prime}$ is an ideal of $B$. Then $I \bowtie^{f} J$ is a weakly prime ideal of $A \bowtie^{f} J$.

Proof. One can easily check that $J=M \subseteq f(A)=B, f(M) J=M^{2}=0$. By [3, Corollary 18], $I$ is a weakly prime of $A$ since $I^{\prime}$ is weakly prime of $B$. So, by Corollary 2.4, I $\bowtie^{f} J$ is weakly prime ideal of $A \bowtie^{f} J$. 
Let $I$ be a proper ideal of $A$. The (amalgamated) duplication of $A$ along $I$ is a special amalgamation given by

$$
A \bowtie I:=A \bowtie^{i d_{A}} I=\{(a, a+i) \mid a \in A, i \in I\} .
$$

The corollary is an immediate consequence of assertion (1) of Theorem 2.1 on the transfer of weakly prime property to duplications.

Corollary 2.6. Let $A$ be a ring, $I$ and $P$ be a proper ideals of $A$. Then $P \bowtie I$ is a weakly prime ideal of $A \bowtie I$ if and only if $P$ is a weakly prime ideal of $A$ and $a, b \in A \backslash P$ with $a b=0$ then $a j+b i+i j=0$ for every $i, j \in I$.

Remark 2.7. Let $f: A \rightarrow B$ be a ring homomorphism and $J$ be an ideal of $B$. Consider $I$ (resp., $H$ ) be an ideal of $A$ (resp., $f(A)+J$ ) such that $f(I) J \subseteq H \subseteq J$. Observe that $I \bowtie^{f} H:=\{(i, f(i)+h) / i \in I, h \in H\}$ is an ideal of $A \bowtie^{f} J$.

By the previous remark, we establish the next result.

Proposition 2.8. Under the above notation. If I $\bowtie^{f} H$ is a weakly prime ideal of $A \bowtie^{f} J$, then $I$ is a weakly prime ideal of $A$.

Proof. Assume that $I \bowtie^{f} H$ is a weakly prime ideal of $A \bowtie^{f} J$. Let $0 \neq a b \in I$ where $a, b \in A$. Then, $(0,0) \neq(a, f(a))(b, f(b)) \in I \bowtie^{f} H$ which is a weakly prime ideal of $A \bowtie^{f} J$. Hence, $(a, f(a)) \in I \bowtie^{f} H$ or $(b, f(b)) \in I \bowtie^{f} H$. Therefore, $a \in I$ or $b \in I$ and so $I$ is a weakly prime ideal of $A$, as desired.

The converse of Proposition 2.8 is not true in general, as explained later in Remark 2.10 in the special case of duplications. The next corollary is an immediate consequence of Proposition 2.8 for duplications:

Corollary 2.9. Let $A$ be a ring and $I, H, J$ are ideals of $A$ such that $I J \subseteq H \subset J$. If $I \bowtie H$ is a weakly prime ideal of $A \bowtie J$, then $I$ is a weakly prime ideal of $A$.

Remark 2.10. In the situation of Corollary $2.9, I$ is a weakly prime does not imply that $I \bowtie H$ is a weakly prime ideal of $A \bowtie J$ in general. Indeed, let $A$ be an integral domain and $H, J$ are nonzero proper ideals of $A$ such that $J^{2} \subseteq H \subset J$ and $J$ is a weakly prime ideal of $A$. Consider the ideal $0 \bowtie H$ of $A \bowtie J$. Notice that 0 is a weakly prime ideal of $A$ as it is a prime ideal. Let $0 \neq j \in J \backslash H$. Then $(0,0) \neq(0, j)(0, j) \in 0 \bowtie H$ but $(0, j) \notin 0 \bowtie H$. Hence, $0 \bowtie H$ is not a weakly prime ideal of $A \bowtie J$. For instance take $A:=\mathbb{Z}, J:=4 \mathbb{Z}, H:=8 \mathbb{Z}$ and $j:=12 \in J$. Obviously 0 is a weakly prime ideal as it is a prime ideal of $A$ and $0 \bowtie H$ is not a weakly prime ideal of $A \bowtie J$.

Unlike to the case of prime ideals of $A \bowtie^{f} J$, the weakly prime ideals need not have the form $P^{\prime} f=P \bowtie^{f} J$, where $P$ is prime ideal of $A$ or $\bar{Q}^{f}=\{(a, f(a)+j) / a \in A, j \in J$ and $f(a)+j \in Q\}$, where $Q$ is prime ideal of $B$ not containing $J$. Indeed, let $K$ be a field, $E$ be a $K$-vector space, $f: K \longrightarrow K \propto E$ and $J=0 \propto E$ be an ideal of $K \propto E$. Hence, $K \bowtie^{f} J$ is quasilocal ring with maximal ideal $M=0 \propto E$ and $M^{2}=0$. Then, every proper ideal of $K \bowtie^{f} J$ is weakly prime ideal. Our next result pursues the study of the ideal-theoretic structure of the amalgamation $A \bowtie^{f} J$.

Proposition 2.11. Let $(A, B)$ be a pair of rings, $f: A \longrightarrow B$ be a ring homomorphism, $J$ be an ideal of $B$ and $H=0 \times K$ be an ideal of $A \bowtie^{f} J$ such that $0 \neq K \subset J$. Then the following statements hold:

(1) Assume that $J$ be regular ideal of $B$. If $0 \times K$ is a weakly prime, then $f^{-1}(J)=0$.

(2) Assume that $A$ is an integral domain. Then $0 \times K$ is weakly prime ideal of $A \bowtie^{f} J$ if and only if $0 \neq i(f(a)+j) \in K$ implies $i \in K$ and $0 \neq i j \in K$ implies $i \in K$ or $j \in K, \forall a \in A-\{0\}$ and $\forall i, j \in J$. 
Proof. (1) Assume by the way of contradiction that $f^{-1}(J) \neq 0$. Pick a nonzero element $a$ of $f^{-1}(J)$ and let $j$ be regular element of $J$. Without loss of generality, we may assume that $j \notin K$. Consider $0 \neq k \in K$, we have $(0,0) \neq(a, k)(0, j) \in 0 \times K$ and neither $(a, k) \in 0 \times K$ nor $(0, j) \in 0 \times K$, the desired contradiction (as $0 \times K$ is a weakly prime ideal of $\left.A \bowtie^{f} J\right)$.

(2) Assume that $A$ is an integral domain and $0 \times K$ is a weakly prime ideal of $A \bowtie^{f} J$. Let $a \in A-\{0\}$ and $i, j \in J$ such that $0 \neq i(f(a)+j) \in K$. Hence, $(0,0) \neq(0, i)(a, f(a)+j) \in$ $0 \times K$. Then $(0, i) \in 0 \times K$ since $(a, f(a)+j) \notin 0 \times K$ and $0 \times K$ is weakly prime ideal of $A \bowtie^{f} J$. Therefore, $i \in K$. Next, if $0 \neq i j \in K$, then $(0,0) \neq(0, i)(0, j) \in 0 \times K$. Since $0 \times K$ is a weakly prime ideal, then $(0, i) \in 0 \times K$ or $(0, j) \in 0 \times K$ and hence $i \in K$ or $j \in K$. Conversely, let $(0,0) \neq(a, f(a)+i)(b, f(b)+j)=(a b, f(a b)+j f(a)+i f(b)+i j) \in 0 \times K$, where $(a, f(a)+i),(b, f(b)+j) \in A \bowtie^{f} J$. Then $a b=0$ implies $a=0$ or $b=0$. Two cases are then possible:

Case 1: $a=b=0$. Then $0 \neq i j \in K$. By hypothesis, it follows $i \in K$ or $j \in K$. Therefore, $(a, f(a)+i)=(0, i) \in 0 \times K$ or $(b, f(b)+j)=(0, j) \in 0 \times K$, as desired.

Case 2: $a \neq 0$ or $b \neq 0$. If $a=0$ and $b \neq 0$. Then $0 \neq i(f(b)+j) \in K$. From assumption, $i \in K$ and so $(0, i) \in 0 \times K$. Similarly, if $a \neq 0$ and $b=0$, we get $(0, j) \in 0 \times K$.

Thus, $0 \times K$ is a weakly prime ideal of $A \bowtie^{f} J$ and this completes the proof of the proposition.

The next theorem gives a characterization of the general form of weakly prime ideals in a particular case of the construction of $A \bowtie^{f} J$.

Theorem 2.12. Let $A$ be a ring such that $a^{n}=0$ implies that $a=0$ for some $n \geq 2$, $B$ be a ring, $f: A \longrightarrow B$ be a ring homomorphism and $J$ be an ideal of $B$ such that $J \subseteq N i l p(B)$. Let $H$ be a nonzero weakly prime ideal of $A \bowtie^{f} J$. Then:

(1) If $H \subseteq 0 \times J$, then $H=0 \times K$, where $K$ is weakly prime subideal of $J$.

(2) If $H \nsubseteq 0 \times J$, then $H=I \bowtie^{f} J$, where $I$ is a weakly prime ideal of $A$.

Before proving Theorem 2.12, we establish the following lemma.

Lemma 2.13. Let $A$ be $a$ ring and $I$ be a weakly prime ideal of $A$. Suppose $a^{n}=0$ for some $n \geq 2$ and $a \in A \backslash I$. Then, $(a+i)^{n}=(a-i)^{n}=0$.

Proof. Let $i \in I$. Notice first that $(a+i)^{n}=a^{n}+i^{n}+\sum_{k=1}^{n-1} C_{n-1}^{k} a^{k} i^{n-1-k}=0+i^{n}+$ $\sum_{k=1}^{n-1} C_{n-1}^{k} a^{k} i^{n-1-k} \in I$. Since $a \in A \backslash I$, then $a+i \notin I$. Now, we claim that $(a+i)^{n}=0$. Deny. $0 \neq(a+i)^{n} \in I$. Using the fact that $I$ is a weakly prime ideal, it follows that $a+i \in I$, which is absurd. Similarly, we have $(a-i)^{n}=0$.

Proof of Theorem 2.12. (1) Straightforward.

(2) Assume that $H \nsubseteq 0 \times J$. Then there exists $(a, f(a)+j) \in H$ such that $a \neq 0$. Set $I=\{b \in A /(b, f(b)+k) \in H$ for some $k \in J\}$. We claim that $H=I \bowtie^{f} J$. It is clear that $H \subseteq I \bowtie^{f} J$. Let $(x, f(x)+h) \in I \bowtie^{f} J$. Two cases are then possible:

Case 1: $x=0$. We may assume that $(0, h) \notin H$. Since $J \subseteq N i l p(B)$, then $(0, h)^{n}=0$ for some $n \geq 2$. Hence, by Lemma 2.13 , it follows that $[(a, f(a)+j)+(0, h)]^{n}=(0,0)$. Then $a^{n}=0$ and so $a=0$, contradiction.

Case 2: $x \neq 0$. Hence, $(x, f(x)+i) \in H$ for some $i \in J$. We claim that $(0, h-i) \in$ $H$. Deny. $(0, h-i)^{n}=0$ for some $n \geq 2$ since $J \subseteq N i l p(B)$. So, by Lemma 2.13, $[(a, f(a)+j)-(0, h-i)]^{n}=0$. Hence $a^{n}=0$ and so $a=0$ by hypothesis, a contradiction. Consequently, $(0, h-i) \in H$. So $(x, f(x)+h)=(x, f(x)+i)+(0, h-i) \in H$.

Therefore, $H=I \bowtie^{f} J$ where $I$ is an ideal of $A$. By assertion (1) of Theorem 2.1, $I$ is a weakly prime ideal of $A$.

Remark 2.14. If we take $A=B, f=i d_{A}$ and $J=I$ be an ideal of $A$. In this situation, the conditions given in Theorem 2.12 force $I$ to be equal to 0 . 
To end this section, we give necessary and sufficient conditions for which each proper ideal of $A \bowtie^{f} J$ is a weakly prime ideal. Notice that if $J=B$, then the amalgamation degenerates in the direct product $A \bowtie^{f} J=A \times B$. If $J=0$, then $A \bowtie^{f} J \simeq A$. Also recall that $f^{-1}(J)=0$ if and only if $A \bowtie^{f} J$ and $f(A)+J$ are isomorphic [9, Proposition 2.1]. Therefore, to avoid the trivial cases $A \bowtie^{f} J=A \times B, A \bowtie^{f} J \simeq A$ and $A \bowtie^{f} J \simeq f(A)+J$, in the next result, we may assume that " $f^{-1}(J) \neq 0$ " and $J$ is "a nonzero proper" ideal of $B$.

Theorem 2.15. Let $f: A \longrightarrow B$ be a ring homomorphism and $J$ be a nonzero proper ideal of $B$. Assume that $f^{-1}(J) \neq 0$. Then $A \bowtie^{f} J$ has every proper ideal weakly prime if and only if $(A, \mathfrak{m})$ is quasilocal ring with $\mathfrak{m}^{2}=0$ and $(f(A)+J, f(\mathfrak{m})+J)$ is a quasilocal ring with $(f(\mathfrak{m})+J)^{2}=0$.

The proof of Theorem 2.15 requires the following lemma.

Lemma 2.16 ([3, Theorem 8]). A commutative ring $R$ has every proper ideal weakly prime if and only if either $(1)(R, \mathfrak{m})$ is quasilocal ring (possibly a field) with $\mathfrak{m}^{2}=0$ or (2) $R=F_{1} \times F_{2}$ where $F_{1}, F_{2}$ are fields.

Proof of Theorem 2.15. Assume that $A \bowtie^{f} J$ has every proper ideal weakly prime. Then by Lemma 2.16, two cases are then possible:

Case 1: $\left(A \bowtie^{f} J, M\right)$ is quasilocal with $M^{2}=0$.

By [8, Proposition $5.1(3)]$, both $(A, \mathfrak{m})$ and $(f(A)+J, f(\mathfrak{m})+J)$ are quasilocal rings. Notice that $f^{-1}(J) \neq A$ (as $J$ is a proper ideal of $B$ ). So, there exists a maximal ideal $\mathfrak{m}$ such that $f^{-1}(J) \subset \mathfrak{m}$. Combining [9, Proposition 2.6 (4)] and [9, Proposition 2.1 (2)], it follows that $\mathfrak{m} \bowtie^{f} J$ is the unique maximal ideal of $A \bowtie^{f} J$. Since $M^{2}=\left(\mathfrak{m} \bowtie^{f} J\right)^{2}=0$, then one can easily check that $\mathfrak{m}^{2}=0$ and $(f(\mathfrak{m})+J)^{2}=0$. Therefore, $A$ and $f(A)+J$ have every proper ideal weakly prime.

Case 2: $A \bowtie^{f} J=F_{1} \times F_{2}$ where $F_{1}, F_{2}$ are fields.

Then $A \bowtie^{f} J=F_{1} \times F_{2} \subseteq A \times B$. Let $0 \neq x \in J$. Then $(0,0) \neq(0, x) \in A \bowtie^{f} J$. It follows that $x$ is invertible, making $J=B$, which is absurd, as $J$ is a proper ideal of $B$.

Conversely, assume that $A$ and $f(A)+J$ are quasilocal rings with $\mathfrak{m}^{2}=0$ and $(f(\mathfrak{m})+J)^{2}=$ 0 . Since $f^{-1}(J) \neq 0$ and $J$ is nonzero proper ideal of $B$, then we may assume $A$ and $f(A)+J$ are not fields. By [11, Corollary 5.5], $\left(A \bowtie^{f} J, M\right)=\left(A \bowtie^{f} J, \mathfrak{m} \bowtie^{f} J\right)$ is quasilocal. The fact that $A$ and $f(A)+J$ have every proper ideal weakly prime, then necessarily $\mathfrak{m}^{2}=0$ and $(f(\mathfrak{m})+J)^{2}=0$ and so $M^{2}=\left(\mathfrak{m} \bowtie^{f} J\right)^{2} \subset(\mathfrak{m} \times(f(\mathfrak{m})+J))^{2}=0$. Hence, $\left(\mathfrak{m} \bowtie^{f} J\right)^{2}=0$, as desired.

\section{Weakly Krull dimension}

In this section, we study the notion of weakly Krull dimension introduced and studied in [14] and considered as generalization of Krull dimension of commutative ring. Recall that the weakly Krull dimension of $R$ (denoted by $w-\operatorname{dim}(R)$ ) is the supremum of the lenghts of all chains of distincts weakly prime ideals of $R$. It is worthwhile to mention that the Krull dimension of amalgamation was studied in [9]. Now, we study the weakly Krull dimension of $A \bowtie^{f} J$. By Theorem 2.12, we know under some hypothesis that the weakly prime ideals of $A \bowtie^{f} J$ have the form $0 \times K$, where $K$ is a subideal of $J$ or $I \bowtie^{f} J$, where $I$ is weakly prime ideal of $A$. Therefore, we proceed our investigation looking for upper bounds of the weakly Krull dimension of $A \bowtie^{f} J$.

Theorem 3.1. Let $A$ be a ring such that $a^{n}=0$ for some $n \geq 2$ implies $a=0$ for each $a \in A, B$ be a ring, $f: A \longrightarrow B$ be a ring homomorphism and $J$ be an ideal of $B$ such that $J \subseteq N i l p(B)$. Then, $w-\operatorname{dim}\left(A \bowtie^{f} J\right) \leq w-\operatorname{dim}(A)+w-\operatorname{dim}(B)$.

Proof. Let $H_{0} \subset H_{1} \subset \cdots \subset H_{n}$ be a chain of weakly prime ideals of $A \bowtie^{f} J$ realizing $w-\operatorname{dim}\left(A \bowtie^{f} J\right)$. By Theorem 2.12, for every $i=0,1, \ldots, n, H_{i}=0 \times K_{i}$, where $K_{i}$ is a 
weakly prime subideal of $J$ or $H_{i}=I_{i} \bowtie^{f} J$, where $I_{i}$ is a weakly prime ideal of $A$. If $0 \times J \subseteq H_{0}$, then each $H_{i}$ has the form $I_{i} \bowtie^{f} J$. So, rewrite the given chain as follows: $I_{0} \bowtie^{f} J \subset I_{1} \bowtie^{f} J \subset \cdots \subset I_{n} \bowtie^{f} J$. The last chain induces a chain of weakly prime ideals of $A$ of length $n$. Therefore $w-\operatorname{dim}\left(A \bowtie^{f} J\right) \leq w-\operatorname{dim}(A)$.

If $0 \times J \nsubseteq H_{n}$. Hence the chain induces a chain of weakly prime ideals of $B$ of length $n$. Therefore, $w-\operatorname{dim}\left(A \bowtie^{f} J\right) \leq w-\operatorname{dim}(B)$.

Finally, we may assume that $m$ the maximum index such that $0 \times J \nsubseteq H_{m}$. According to the form of $H_{i}$ rewrite the given chain as follows:

$$
0 \times K_{0} \subset 0 \times K_{1} \subset \cdots \subset 0 \times K_{m} \subset I_{m+1} \bowtie^{f} J \subset \cdots \subset I_{n} \bowtie^{f} J
$$

where, $K_{0} \subset K_{1} \subset \cdots \subset K_{m}$ is an increasing chain of weakly prime ideals of $B$ and $I_{m+1} \subset \cdots \subset I_{n}$ is an increasing chain of weakly prime ideals of $A$. Hence, $w-\operatorname{dim}\left(A \bowtie^{f}\right.$ $J) \leq w-\operatorname{dim}(A)+w-\operatorname{dim}(B)$, as desired.

Remark 3.2. $\quad$ a) Recall that if $J$ is contained in the nilradical of $B$, then $\operatorname{dim}(U)=$ -1 (where $U=\operatorname{spec}(B) \backslash V(J)$ and conventionally, we set $\operatorname{dim}(\emptyset)=-1$ ). In this case, $\operatorname{dim}(A)=\operatorname{dim}\left(A \bowtie^{f} J\right)$, for more details see [9, Remark 4.5].

b) Note that, if $J=B$ then $A \bowtie^{f} J=A \times B$. In this case, we have w-dim( $\left(A \bowtie^{f}\right.$ $J)=\max \{w-\operatorname{dim}(A), w-\operatorname{dim}(B)\}$ (see [14, Theorem 3.3]).

c) If $f^{-1}(J)=0$, we have $A \bowtie^{f} J \cong f(A)+J$. In this case, w-dim $\left(A \bowtie^{f} J\right)=\mathrm{w}$ $\operatorname{dim}(f(A)+J)$.

d) Note that, if $f^{-1}(J) \neq 0$, then $A \bowtie^{f} J$ is not an integral domain (see [8, Proposition 5.2]). Hence $w-\operatorname{dim}\left(A \bowtie^{f} J\right) \geq \operatorname{dim}\left(A \bowtie^{f} J\right)+1>\operatorname{dim}\left(A \bowtie^{f} J\right)$ since each prime ideal is a weakly prime and 0 is always weakly prime, but in this case is not a prime ideal.

We close this section by giving an illustrative example for Theorem 3.1.

Example 3.3. Let $A=\mathbb{Z}, B=\mathbb{Z} \propto \mathbb{Z} / 4 \mathbb{Z}$ be a trivial ring extension of $\mathbb{Z}$ by the $\mathbb{Z}$-module $\mathbb{Z} / 4 \mathbb{Z}, J=0 \propto \mathbb{Z} / 4 \mathbb{Z}$ be an ideal of $B$ and $f$ be the canonical embedding. Clearly, $J \subseteq N i l p(B)$ and $a^{n}=0$ for some $n \geq 2$ implies $a=0$ for each $a \in A$ since $A$ is integral domain. Then, by application to Theorem 3.1, it follows that w-dim $\left(A \bowtie^{f} J\right)=\mathrm{w}-$ $\operatorname{dim}(B) \leq \mathrm{w}-\operatorname{dim}(A)+\mathrm{w}-\operatorname{dim}(B)$.

Acknowledgment. The authors would like to express their sincere thanks to the anonymous referee for his/her helpful suggestions and comments.

\section{References}

[1] A.G. Agargun, D.D. Anderson and S. Valdes-Leon, Unique factorization ring with zero divisors, Comm. Algebra 27, 1967-1974, 1999.

[2] K. Alaoui Ismaili and N. Mahdou, Coherence in amalgamated algebra along an ideal, Bull. Iranian Math. Soc. 41, 625-632, 2015.

[3] D.D. Anderson and E. Smith, Weakly prime ideals, Houston J. Math. 29 (4), 831-840, 2003.

[4] D.D. Anderson and M. Winders, Idealization of a module, J. Commut. Algebra 1 (1), 3-56, 2009.

[5] A. Badawi, On weakly semiprime ideals of commutative rings, Beitr. Algebra Geom. 51 (4), 1163-1173, 2014.

[6] M. Boisen and P.B. Sheldon, CPI-extensions : overrings of integral domains with special prime spectrums, Canad. J. Math. 29, 722-737, 1977.

[7] M. Chhiti, M. Jarrar, S. Kabbaj and N. Mahdou, Prüfer-like conditions in the amalgamated duplication of a ring along an ideal, Comm. Algebra 43 (1), 249-261, 2015. 
[8] M. D'Anna, C.A. Finocchiaro and M. Fontana, Amalgamated algebras along an ideal, Commutative Algebra and Its Applications, Walter de Gruyter, Berlin, 241-252, 2009.

[9] M. D'Anna, C.A. Finocchiaro and M. Fontana, Properties of chains of prime ideals in an amalgamated algebra along an ideal, J. Pure Appl. Algebra, 1633-1641, 2010.

[10] M. D'Anna and M. Fontana, The amalgamated duplication of ring along an ideal: the basic properties, J. Algebra Appl. 6 (3), 241-252, 2007.

[11] S. Kabbaj, K. Louartiti and M. Tamekkante, Bi-amalgamated algebras along ideals, J. Commut. Algebra 9 (1), 65-87, 2017.

[12] S. Kabbaj, N. Mahdou and M. A. S. Moutui, Bi-amalgamations subject to the arithmetical property, J. Algebra Appl. 16 (2), 11 pages, 2017.

[13] N. Mahdou and M.A.S. Moutui, On (A)-rings and strong (A)-rings issued from amalgamations, Stud. Sci. Math. Hung. 55 (2), 270-279, 2018.

[14] N. Mahdou and Y. Zahir, On weakly prime and weakly semiprime ideals, submitted. 